\title{
Scaling theory of magnetic ordering in the Kondo lattices with anisotropic exchange interactions
}

\author{
V.Yu. Irkhin* and M.I. Katsnelson \\ Institute of Metal Physics, 620219 Ekaterinburg, Russia
}

\begin{abstract}
The lowest-order scaling consideration of the magnetic state formation in the Kondo lattices is performed within the $s-f$ model with inclusion of anisotropy for both the $f-f$ coupling and $s-f$ exchange interaction. The Kondo renormalizations of the effective transverse and longitudinal $s-f$ coupling parameters, spin-wave frequency, gap in the magnon spectrum and ordered moment are calculated in the case of both ferro- and antiferromagnets. The anisotropy-driven change of the scaling behavior (e.g., critical value of $g$ for entering the strong-coupling region and the corresponding critical exponents) is investigated numerically for $N=2$ and analytically in the large- $N$ limit. The dependence of the effective Kondo temperature on the bare $s-f$ coupling parameter $g$ weakens in the presence of anisotropy. The relative anisotropy parameters for both the $s-f$ and $f-f$ coupling are demonstrated to decrease during the renormalization process. The role of next-nearest exchange interactions for this effect in the antiferromagnet is discussed.
\end{abstract}

Typeset using REVTEX 


\section{INTRODUCTION}

Anomalous rare earth and actinide compounds, including so-called Kondo lattices and heavy-fermion systems, are studied extensively by both experimentalists and theorists [2] [6]. It is now a common point of view that the most interesting peculiarities of electronic and magnetic propeties of these systems are due to the interplay of the on-site Kondo effect and intersite magnetic interactions. Whereas the one-impurity Kondo problem, being itself very difficult and rich, is now studied in detail [7], the Kondo-lattice problem is still a subject of many investigations [ [6, 6], Usually this problem is studied within the standard $s-f$ exchange or Anderson models. On the other hand, strong effects of crystal field and anisotropic interactions are expected in anomalous $4 f$ and $5 f$-systems (see, e.g., [9]). These effects can lead to anisotropic terms in the Hamiltonian. It is well known that the change of symmetry of the $s-f$ exchange interaction modifies qualitatively the infrared behavior in the one-impurity case [10,[4,11]. Thus one could expect that similar effects should take place in the lattice case. Therefore a question arises whether anisotropic contributions are important also in the problem of competition of the Kondo effect and magnetism. It should be noted that this question is relevant not only for magnetic systems, but also for models with pseudospin degrees of freedom (e.g., for strongly anharmonic crystals demonstrating band Jahn-Teller effect [12]).

Theoretical investigations of the Kondo-lattice problem use as a rule methods appropriate for calculating low-temperature properties in the strong-couping regime $(1 / N$-expansion [13], slave-boson technique). However, these methods are not convenient for the description of the transition to the weak-coupling regime (in particular, even derivation of the standard Kondo logaruithms is here a non-trivial problem [14]). In our previous paper [8] we have proposed an alternative approach which starts from the weak-coupling regime and is based on summing up leading divergent terms by the renormalization group method. We have investigated the formation of magnetic state in the periodic $s-f$ exchange and Coqblin-Schrieffer models

with the $f$-subsystem being described by the isotropic Heisenberg Hamiltonian. The aim of 
the present paper is the investigation of formation of the magnetic Kondo-lattice state for various magnetic phases with account of the anisotropy in both the localized-spin subsystem and $s-f$ exchange interaction.

In Sect.2 we discuss the theoretical model and calculate the logarithmic Kondo corrections to the spin-wave spectrum of anisotropic metallic ferro- and antiferromagnets. In Sect.3 we derive the lowest-order scaling equations for the effective transverse and longitudinal $s-f$ exchange parameters and renormalized magnon frequencies. In Sect.4 we present a simple analytical solution with magnon spectrum renormalizations being neglected, which is possible in the large- $N$ limit of the Coqblin-Schrieffer model. In Sect.5 we discuss results of the numerical solution of the full scaling equations for $N=2$ in the presence of the anisotropy in localized-spin system only and investigate new features which occur in comparison with the isotropic case. In Sect.6, influence of the anisotropic $s-f$ coupling is considered.

\section{THEORETICAL MODELS AND KONDO CORRECTIONS TO THE SPECTRUM OF SPIN EXCITATIONS}

To treat the Kondo effect in a lattice we use the $s-d(f)$ exchange model

$$
H=\sum_{\mathbf{k} \sigma} t_{\mathbf{k}} c_{\mathbf{k} \sigma}^{\dagger} c_{\mathbf{k} \sigma}+H_{f}+H_{s f}=H_{0}+H_{s f}
$$

where $t_{\mathbf{k}}$ is the band energy. We consider the pure spin $s-d(f)$ exchange Hamiltonian with

$$
\begin{aligned}
H_{f} & =\sum_{\mathbf{q}} J_{\mathbf{q}} \mathbf{S}_{-\mathbf{q}} \mathbf{S}_{\mathbf{q}}+\eta \sum_{\mathbf{q}} J_{\mathbf{q}} S_{-\mathbf{q}}^{z} S_{\mathbf{q}}^{z}-K \sum_{i}\left(S_{i}^{z}\right)^{2}, \\
H_{s f} & =-\sum_{\mathbf{k} \mathbf{k}^{\prime} \alpha \beta}\left[I_{\|} S_{\mathbf{k}-\mathbf{k}^{\prime}}^{z}\left(c_{\mathbf{k} \uparrow}^{\dagger} c_{\mathbf{k}^{\prime} \uparrow}-c_{\mathbf{k} \downarrow}^{\dagger} c_{\mathbf{k}^{\prime} \downarrow}\right)+I_{\perp}\left(S_{\mathbf{k}-\mathbf{k}^{\prime}}^{+} c_{\mathbf{k} \downarrow}^{\dagger} c_{\mathbf{k}^{\prime} \uparrow}+S_{\mathbf{k}-\mathbf{k}^{\prime}}^{-} c_{\mathbf{k} \uparrow}^{\dagger} c_{\mathbf{k}^{\prime} \uparrow}\right)\right]
\end{aligned}
$$

where $\mathbf{S}_{i}$ and $\mathbf{S}_{\mathbf{q}}$ are spin operators and their Fourier transforms, $\eta>0$ and $K>0$ are the parameters of the two-site and single-site easy-axis magnetic anisotropy in the $f$-subsystem, respectively. Note that our consideration can be formally generalized on the Coqblin-Schrieffer

model with arbitrary $N$ (cf. Ref. [8]) or to a more general form of the $s-f$ coupling parameter matrix [1]. For simplicity, we neglect $\mathbf{k}$-dependence of the $s-f$ parameter which 
occurs in the degenerate $s-f$ model due to the angle dependence of the coupling (see Ref. [8]). Of course, in fact the $f-f$ exchange has usually the Ruderman-Kittel-Kasuya-Yosida (RKKY) origin and is determined by the same $s-f$ coupling. Thus, generally speaking, the anisotropy of the $s-f$ coupling and $f$-subsystem are not independent. However, crystal-field effects are known to be more important in formation of magnetic anisotropy in rare-earth metals than anisotropic exchange interactions [17 (in this case, the anisotropic $s-f$ coupling is obtained by expansion in the parameter $k_{F} r_{f}, r_{f}$ being the $f$-shell radius, and contains, unlike (3), terms of the type $\left.(\mathbf{k S})\left(\mathbf{k}^{\prime} \mathbf{S}\right)\right)$. On the other hand, the situation, where anisotropy occurs in the $s-f$ coupling only, may be also considered: this corresponds to the strong "direct" $f-f$ exchange (e.g., superexchange) interaction which is characteristic for some $f$-compounds. In the Coqblin-Schrieffer model, which is more appropriate for cerium compounds, crystal field results in occurrence of anisotropic $s-f$ coupling [15, 16] and new excitation branches [9,8]. For simplicity, we restrict ourselves to treatment of a single magnon mode in the simplest $s-f$ model (11).

In the ferromagnetic (FM) state the spin-wave spectrum for the Hamiltonian (2) reads

$$
\begin{aligned}
\omega_{\mathbf{q}} & =\omega_{0}+\omega_{e x}(\mathbf{q}), \\
\omega_{e x}(\mathbf{q}) & =2 S\left(J_{\mathbf{q}}-J_{0}\right), \omega_{0}=-2 S \eta J_{0}+(2 S-1) K
\end{aligned}
$$

To find the Kondo logarithmic corrections to the spin-wave spectrum we calculate the magnon Green's functions in the model (11). For a ferromagnet we obtain to second order in $I$ (cf. the calculations in the isotropic case [18])

$$
\begin{aligned}
\left\langle\left\langle b_{\mathbf{q}} \mid b_{\mathbf{q}}^{\dagger}\right\rangle\right\rangle_{\omega}=[\omega & -\omega_{\mathbf{q}}-2 \sum_{\mathbf{p}}\left(J_{0}+J_{\mathbf{q}-\mathbf{p}}-J_{\mathbf{p}}-J_{\mathbf{q}}+\omega_{0} / 2 S\right)\left\langle b_{\mathbf{p}}^{\dagger} b_{\mathbf{p}}\right\rangle \\
& \left.-2 S \sum_{\mathbf{k}}\left(I_{\perp}^{2} \frac{n_{\mathbf{k}}-n_{\mathbf{k}-\mathbf{q}}}{\omega+t_{\mathbf{k}}-t_{\mathbf{k}-\mathbf{q}}}-I_{\|}^{2} \frac{\partial n_{\mathbf{k}}}{\partial t_{\mathbf{k}}}\right)-2 \sum_{\mathbf{p}}\left(I_{\|}^{2} \phi_{\mathbf{p q} \omega}-I_{\perp}^{2} \phi_{\mathbf{p} 00}\right)\right]^{-1}
\end{aligned}
$$

where we have taken into account kinematic requirements in the magnon anharmonicity terms by introducing the factor of $(2 S-1) / 2 S$ at $K$ (this replacement may be justified by considering higher-order terms in the formal parameter $1 / S), n_{\mathbf{k}}=n\left(t_{\mathbf{k}}\right)$ is the Fermi function, 


$$
\phi_{\mathbf{p q} \omega}=\sum_{\mathbf{k}} \frac{n_{\mathbf{k}}\left(1-n_{\mathbf{k}+\mathbf{p}-\mathbf{q}}\right)}{\omega+t_{\mathbf{k}}-t_{\mathbf{k}+\mathbf{p}-\mathbf{q}}-\omega_{\mathbf{p}}}
$$

The averages that enter (6) can be obtained from the spectral representation for the Green's function (6) to first order in $1 / S$ and contain the singular contributions

$$
\delta\left\langle b_{\mathbf{q}}^{\dagger} b_{\mathbf{q}}\right\rangle=S I_{\perp}^{2} \Phi_{\mathbf{q}}
$$

with

$$
\Phi_{\mathbf{q}}^{F M}=\sum_{\mathbf{k}} \frac{n_{\mathbf{k}}\left(1-n_{\mathbf{k}+\mathbf{q}}\right)}{\left(t_{\mathbf{k}}-t_{\mathbf{k}+\mathbf{q}}-\omega_{\mathbf{q}}\right)^{2}}
$$

Expanding the denominators of (7) in the magnon frequencies we obtain the singular correction to the pole of the magnon Green's function

$$
\delta \omega_{\mathbf{q}}=-2 S\left(I_{\perp}^{2}+I_{\|}^{2}\right) \sum_{\mathbf{p}}\left(J_{\mathbf{p}}-J_{\mathbf{q}-\mathbf{p}}+J_{\mathbf{q}}-J_{0}+\omega_{0} / 2 S\right) \Phi_{\mathbf{p}}^{F M}
$$

This result can be represented as

$$
\begin{aligned}
\delta \omega_{e x}(\mathbf{q}) / \omega_{e x}(\mathbf{q}) & =-\left(I_{\perp}^{2}+I_{\|}^{2}\right)\left(1-\alpha_{\mathbf{q}}\right) \sum_{\mathbf{p}} \Phi_{\mathbf{p}}^{F M} \\
\delta \omega_{0} / \omega_{0} & =-\left(I_{\perp}^{2}+I_{\|}^{2}\right) \sum_{\mathbf{p}} \Phi_{\mathbf{p}}^{F M}
\end{aligned}
$$

where $0<\alpha_{\mathbf{q}}<1$. Passing into real space (see [8]) yields

$$
\alpha_{\mathbf{q}}=\sum_{\mathbf{R}} J_{\mathbf{R}}\left|\left\langle e^{i \mathbf{k R}}\right\rangle_{t_{\mathbf{k}}=E_{F}}\right|^{2}[1-\cos \mathbf{q} \mathbf{R}] / \sum_{\mathbf{R}} J_{\mathbf{R}}[1-\cos \mathbf{q} \mathbf{R}]
$$

In the approximation of nearest neighbors at the distance $d$, the quantity $\alpha$ does not depend on q. For a spherical Fermi surface we have

$$
\alpha_{\mathbf{q}}=\alpha=\left|\left\langle e^{i \mathbf{k R}}\right\rangle_{t_{\mathbf{k}}=E_{F}}\right|^{2}=\left(\frac{\sin k_{F} d}{k_{F} d}\right)^{2}
$$

Hereafter we put $\alpha=$ const. Then we may use in further consideration of the scaling equations a single renormalization parameter, rather than the whole function of $\mathbf{q}$.

Now we consider a two-sublattice antiferromagnet (AFM) with the wavevector of magnetic structure $\mathbf{Q}$, 


$$
\left\langle S_{i}^{z}\right\rangle=S \cos \mathbf{Q R}_{i},\left\langle S_{i}^{y}\right\rangle=\left\langle S_{i}^{x}\right\rangle=0
$$

$\left(J_{\mathbf{Q}}=J_{\min }<0 ; 2 \mathbf{Q}\right.$ is equal to a reciprocal lattice vector, so that $\cos ^{2} \mathbf{Q} \mathbf{R}_{i}=1, \sin ^{2} \mathbf{Q} \mathbf{R}_{i}=$ 0 ; only in this case we can retain the definitions of $I_{\perp}$ and $I_{\|}$in the local coordinate system). Passing to the spin-deviation operators in the local coordinate system where

$$
S_{i}^{z}=\hat{S}_{i}^{z} \cos \mathbf{Q R}_{i}, S_{i}^{y}=\hat{S}_{i}^{y} \cos \mathbf{Q} \mathbf{R}_{i}, S_{i}^{x}=\hat{S}_{i}^{x}
$$

we derive

$$
\begin{aligned}
& H_{f}=\text { const }+\sum_{\mathbf{q}}\left[C_{\mathbf{q}} b_{\mathbf{q}}^{\dagger} b_{\mathbf{q}}+\frac{1}{2} D_{\mathbf{q}}\left(b_{-\mathbf{q}} b_{\mathbf{q}}+b_{\mathbf{q}}^{\dagger} b_{-\mathbf{q}}^{\dagger}\right)\right]+\ldots \\
& C_{\mathbf{q}}=S\left(J_{\mathbf{Q}+\mathbf{q}}+J_{\mathbf{q}}-2 J_{\mathbf{Q}}(1+\eta)\right)+(2 S-1) K, D_{\mathbf{q}}=S\left(J_{\mathbf{q}}-J_{\mathbf{Q}+\mathbf{q}}\right)
\end{aligned}
$$

Diagonalizing (15) we obtain the spin-wave spectrum

$$
\begin{aligned}
\omega_{\mathbf{q}}^{2} & =C_{\mathbf{q}}^{2}-D_{\mathbf{q}}^{2} \simeq \omega_{0}^{2}+\omega_{e x}^{2}(\mathbf{q}) \\
\omega_{e x}(\mathbf{q}) & =2 S\left(J_{\mathbf{q}}-J_{\mathbf{Q}}\right)^{1 / 2}\left(J_{\mathbf{Q}+\mathbf{q}}-J_{\mathbf{Q}}\right)^{1 / 2}, \\
\omega_{0}^{2} & =2 S\left(J_{0}-J_{\mathbf{Q}}\right)\left[(2 S-1) K-2 S \eta J_{\mathbf{Q}}\right]
\end{aligned}
$$

where we have neglected a weak wavevector dependence of $\omega_{0}$.

The Kondo correction to the spectrum (17) reads (cf. Refs. [8,19])

$$
\begin{aligned}
\delta \omega_{\mathbf{q}}^{2}= & -2 \sum_{\mathbf{p}}\left[I_{\perp}^{2} \omega_{\mathbf{q}}^{2}+2 S^{2} I_{\perp}^{2}\left(J_{\mathbf{Q}+\mathbf{q}}+J_{\mathbf{q}}-2 J_{\mathbf{Q}}\right)\left(J_{\mathbf{p}}+J_{\mathbf{Q}+\mathbf{p}}-J_{\mathbf{Q}+\mathbf{q}-\mathbf{p}}-J_{\mathbf{q}-\mathbf{p}}\right)\right. \\
& \left.+2\left(I_{\|}^{2}-I_{\perp}^{2}\right)\left(C_{\mathbf{q}} C_{\mathbf{p}-\mathbf{q}}-D_{\mathbf{q}} D_{\mathbf{p}-\mathbf{q}}\right)\right] \Phi_{\mathbf{p}}^{A F M}
\end{aligned}
$$

with

$$
\Phi_{\mathbf{q}}^{A F M}=\sum_{\mathbf{k}} \frac{n_{\mathbf{k}}\left(1-n_{\mathbf{k}+\mathbf{q}}\right)}{\left(t_{\mathbf{k}}-t_{\mathbf{k}+\mathbf{q}}\right)^{2}-\omega_{\mathbf{q}}^{2}}
$$

For an antiferromagnet in the nearest-neighbor approximation $\left(J_{\mathbf{Q}+\mathbf{q}}=-J_{\mathbf{q}}\right)$ we obtain from (20)

$$
\begin{aligned}
\delta \omega_{e x}^{2}(\mathbf{q}) / \omega_{e x}^{2}(\mathbf{q}) & =-2\left[I_{\perp}^{2}-\alpha\left(I_{\|}^{2}-I_{\perp}^{2}\right)\right] \sum_{\mathbf{p}} \Phi_{\mathbf{p}}^{A F M} \\
\delta \omega_{0}^{2} / \omega_{0}^{2} & =-2\left[I_{\perp}^{2}-(1-\alpha) s\left(I_{\|}^{2}-I_{\perp}^{2}\right)\right] \sum_{\mathbf{p}} \Phi_{\mathbf{p}}^{A F M}
\end{aligned}
$$


where $s=4 S^{2} J_{\mathbf{Q}}^{2} / \omega_{0}^{2} \gg 1$.

Introducing next-nearest-neighbor interactions and putting for simplicity $I_{\|}=I_{\perp}=I$ we obtain

$$
\delta \omega_{\mathbf{q}}^{2}=-2\left[\omega_{\mathbf{q}}^{2}-8 S^{2} \alpha_{\mathbf{q}}^{(2)}\left(J_{\mathbf{q}}^{(2)}-J_{\mathbf{Q}}\right)\left(J_{\mathbf{q}}^{(2)}-J_{\mathbf{Q}}^{(2)}\right)\right] \sum_{\mathbf{p}} \Phi_{\mathbf{p}}^{A F M}
$$

where

$$
J_{\mathbf{q}}^{(1,2)}=\frac{1}{2}\left(J_{\mathbf{q}} \mp J_{\mathbf{q}+\mathbf{Q}}\right)
$$

correspond to the contribution of nearest and next-nearest neighbors, and $\alpha_{\mathbf{q}}^{(2)}$ is given (12) with the sum over the next-nearest neighbors. Provided that next-nearest neighbor exchange interaction is ferromagnetic (so that the AFM ordering is stable), $J_{\mathbf{q}}^{(2)}-J_{\mathbf{Q}}^{(2)}>0$ and the next-nearest neighbors result in decreasing the Kondo suppression of the magnon frequency, as well as nearest neighbors in the FM case. Then, instead of (22), we can use phenomenologically (e.g., in the long-wave limit) the expression

$$
\begin{aligned}
\delta \omega_{e x}(\mathbf{q}) / \omega_{e x}(\mathbf{q}) & =-\left(1-\alpha^{\prime}\right) I^{2} \sum_{\mathbf{p}} \Phi_{\mathbf{p}}^{A F M} \\
\delta \omega_{0} / \omega_{0} & =-I^{2} \sum_{\mathbf{p}} \Phi_{\mathbf{p}}^{A F M}
\end{aligned}
$$

with $\alpha^{\prime} \propto \alpha^{(2)} J^{(2)} / J^{(1)}$. In the opposite case of AFM next-nearest neighbor exchange the situation is more complicated. In particular, the simple collinear antiferromagnetism can become unstable, and formation of the spiral structure is possible. Note the difference between the FM and AFM cases by a factor of 2, which is due to violation of time-reversal symmetry in a ferromagnet (terms that are linear in $\omega$ give a contribution).

The quantities (9), (21) determine also the singular correction to the (sublattice) magnetization

$$
\delta \bar{S} / S=-\frac{1}{S} \sum_{\mathbf{q}} \delta\left\langle b_{\mathbf{q}}^{\dagger} b_{\mathbf{q}}\right\rangle=-I_{\perp}^{2} \sum_{\mathbf{q}} \Phi_{\mathbf{q}}^{F M, A F M}
$$




\section{SCALING EQUATIONS}

Using the perturbation theory results we can write down the system of scaling equations in the case of the Kondo lattice for various magnetic phases. Their derivation in the isotropic case is described in detail in Ref. [8]. As well as in this paper, we apply the "poor man scaling" approach [20]. In this method one considers the dependence of effective (renormalized) model parameters on the cutoff parameter $C(-D<C<0$, here and hereafter the energy is calculated from the Fermi energy $E_{F}=0$ ) which occurs at picking out the Kondo singular terms.

The renormalization of $I_{\|}$is obtained from renormalization of the magnetic splitting in

electron spectrum, and of $I_{\perp}$ from renormalization of the second-order contribution to the electron self-energy (see corresponding perturbation expressions for a ferromagnet in Ref. [21]). The renormalized $I_{\perp}$ chosen in such a way coincides with the three-leg vertex (with two electron lines with opposite spins and one magnon line) which yields the most natural definition in a magnetically ordered case and agrees with the one-impurity scaling consideration [7] To find the equation for the effective coupling parameters $I_{e f}^{\alpha}(C)\left(I_{e f}^{\alpha}(-D)=I_{e f}^{\alpha}\right)$ we have to calculate the contribution of intermediate electron states near the Fermi level with $C<t_{\mathbf{k}+\mathbf{q}}<C+\delta C$ in the sums that enter expressions for the self-energies (which include, unlike Ref. [8], magnon frequencies with a gap). Then we obtain

$$
\begin{aligned}
& \delta I_{e f}^{\|}(C)=2 \rho I_{\perp}^{2} \eta\left(-\frac{\bar{\omega}_{e x}}{C},-\frac{\omega_{0}}{C}\right) \delta C / C \\
& \delta I_{e f}^{\perp}(C)=2 \rho I_{\perp} I_{\|} \eta\left(-\frac{\bar{\omega}_{e x}}{C},-\frac{\omega_{0}}{C}\right) \delta C / C
\end{aligned}
$$

where $\rho$ is the density of states at the Fermi level, $\bar{\omega}_{e x}$ is a characteristic spin-fluctuation energy, $\omega_{0}$ is the gap in the spin-wave spectrum, $\eta(x)$ is a scaling function which satisfies the condition $\eta(0)=1$ which guarantees the correct one-impurity limit. For both FM and AFM phases we have

$$
\eta^{F M, A F M}\left(-\frac{\bar{\omega}_{e x}}{C},-\frac{\omega_{0}}{C}\right)=\left\langle\left(1-\omega_{\mathbf{k}-\mathbf{k}^{\prime}}^{2} / C^{2}\right)^{-1}\right\rangle_{t_{k}=t_{k^{\prime}}=E_{F}}
$$


where the magnon frequencies are given by (4), (17). The corresponding analytical expressions are presented in Appendix.

The $C$-dependent renormalizations of the spin-wave frequencies and ground-state moment are obtained in the same way as in the isotropic case [8] from (10), (20) (22), (24), (25) and expressed in terms of the same scaling functions. Introducing the dimensionless coupling constants

$$
g_{e f}^{\alpha}(C)=-2 \rho I_{e f}^{\alpha}(C), g_{\alpha}=-2 \rho I_{\alpha}
$$

(we will drop sometimes the index $\|$, but not $\perp$, so that $g_{e f}(C) \equiv g_{e f}^{\|}(C)$ ) and replacing $g_{\alpha} \rightarrow g_{e f}^{\alpha}(C), \bar{\omega}_{e x} \rightarrow \bar{\omega}_{e x}(C), \omega_{0} \rightarrow \omega_{0}(C)$ in the right-hand parts of (26) and expressions for $\delta \bar{\omega}_{e x}(C), \delta \omega_{0}(C)$ and $\delta \bar{S}_{e f}(C)$, we obtain the system of scaling equation

$$
\begin{gathered}
\partial g_{e f}^{\|}(C) / \partial C=-\left[g_{e f}^{\perp}(C)\right]^{2} \Lambda \\
\partial g_{e f}^{\perp}(C) / \partial C=-g_{e f}^{\|}(C) g_{e f}^{\perp}(C) \Lambda \\
\partial \ln \bar{\omega}_{e x}(C) / \partial C=a \Lambda / 2 \times\left\{\begin{array}{l}
\left\{\left[g_{e f}^{\|}(C)\right]^{2}+\left[g_{e f}^{\perp}(C)\right]^{2}\right\} / 2 \\
{\left[g_{e f}^{\perp}(C)\right]^{2}-\alpha\left\{\left[g_{e f}^{\|}(C)\right]^{2}-\left[g_{e f}^{\perp}(C)\right]^{2}\right\}}
\end{array} \quad \mathrm{AFM}\right. \\
\partial \ln \omega_{0}(C) / \partial C=b \Lambda / 2 \times \begin{cases}\left\{\left[g_{e f}^{\|}(C)\right]^{2}+\left[g_{e f}^{\perp}(C)\right]^{2}\right\} / 2 & \mathrm{FM} \\
{\left[g_{e f}^{\perp}(C)\right]^{2}-s(1-\alpha)\left\{\left[g_{e f}^{\|}(C)\right]^{2}-\left[g_{e f}^{\perp}(C)\right]\right\}} & \mathrm{AFM}\end{cases} \\
\partial \ln \bar{S}_{e f}(C) / \partial C=\left[g_{e f}^{\perp}(C)\right]^{2} \Lambda / 2
\end{gathered}
$$

where

$$
\Lambda=\Lambda\left(C, \bar{\omega}_{e x}(C), \omega_{0}(C)\right)=\eta\left(-\bar{\omega}_{e f}(C) / C,-\omega_{0}(C) / C\right) / C
$$

and

$$
a=\left\{\begin{array}{cc}
2(1-\alpha) & \mathrm{FM} \\
1-\alpha^{\prime} & \mathrm{AFM}
\end{array}, b=\left\{\begin{array}{cc}
2 & \mathrm{FM} \\
1 & \mathrm{AFM}
\end{array}\right.\right.
$$

The integral of motion of the system (28), (29) reads

$$
\left[g_{e f}^{\|}(C)\right]^{2}-\left[g_{e f}^{\perp}(C)\right]^{2}=\mu^{2}=g_{\|}^{2}-g_{\perp}^{2}=\mathrm{const}
$$


so that the equation (28) takes the form

$$
\partial g_{e f}(C) / \partial C=-\left[g_{e f}^{2}(C)-\mu^{2}\right] \Lambda
$$

\section{ANALYTICAL SOLUTION IN THE LARGE- $N$ LIMIT}

In the formal large- $N$ limit in the Coqblin-Schrieffer model where $2 \rightarrow N$ in Eqs.(30)(32)) we can neglect renormalizations of magnon frequencies (note that the true large- $N$ limit in the FM case is somewhat different since symmetry of spin-up and spin-down state contributions is violated for $N>2$, see Ref. [8]). Note that the same approximation is valid for $N=2$ provided that $g$ is well below the critical value $g_{c}$ for entering the strong-coupling region. Then, on taking into account (A1), (A4), equation (36) can be integrated analytically to obtain

$$
\begin{gathered}
\frac{1}{\mu}\left[\operatorname{arctanh}\left(\mu / g_{e f}(C)\right)-\operatorname{arctanh}(\mu / g)\right]=G(C)=-\int_{-D}^{C} \frac{d C^{\prime}}{C^{\prime}} \eta\left(-\frac{\bar{\omega}}{C^{\prime}},-\frac{\omega_{0}}{C^{\prime}}\right) \\
G^{F M}(C)=\ln |C / D|-((1+w) / 2)[(C / \bar{\omega}-1) \ln |1-\bar{\omega} / C|-(C / \bar{\omega}+1) \ln |1+\bar{\omega} / C|] \\
+(w / 2)\left[\left(C / \omega_{0}-1\right) \ln \left|1-\omega_{0} / C\right|-\left(C / \omega_{0}+1\right) \ln \left|1+\omega_{0} / C\right|\right]-1 \\
G_{d=3}^{A F M}(C)=\ln |C / D|-\frac{1}{2}\left[\left(1+w^{2}\right)\left(C^{2} / \bar{\omega}^{2}-1\right) \ln \left|1-\bar{\omega}^{2} / C^{2}\right|\right. \\
\left.-w^{2}\left(C^{2} / \omega_{0}^{2}-1\right) \ln \left|1-\omega_{0}^{2} / C^{2}\right|+1\right] \\
G_{d=2}^{A F M}(C)=\left[\theta\left(C^{2}-\bar{\omega}^{2}\right)+\theta\left(\omega_{0}^{2}-C^{2}\right)\right] \ln \left(\frac{1}{2}\left(\sqrt{\left|C^{2}-\omega_{0}^{2}\right|}+\sqrt{\left|C^{2}-\bar{\omega}^{2}\right|}\right) / D\right) \\
+\theta\left(C^{2}-\omega_{0}^{2}\right) \theta\left(\bar{\omega}^{2}-C^{2}\right) \ln \left(\bar{\omega}_{e x} / 2 D\right)
\end{gathered}
$$

where

$$
\bar{\omega}=\left\{\begin{array}{cc}
\omega_{0}+\bar{\omega}_{e x} & \mathrm{FM} \\
\sqrt{\omega_{0}^{2}+\bar{\omega}_{e x}^{2}} & \mathrm{AFM}
\end{array}\right.
$$

The scaling trajectories described by (37)-(40) are shown in Fig.1 for $\mu \neq 0, w=0$ and in Fig. 2 for $w \neq 0, \mu=0$. Note that these pictures describe adequately the case $N=2$, since $g=0.15$ is considerably lower than the critical values. 
The anisotropy of $s-f$ coupling results in that the dependence $1 / g_{e f}(\xi)$ becomes nonlinear at small $\xi=\ln |D / C|$ where the one-impurity behavior takes place

$$
1 / g_{e f}(\xi) \simeq \mu \tanh [\operatorname{arctanh}(\mu / g)-\mu \xi]
$$

However, this non-linearity is not too strong even for $\mu / g=2 / 3$ (Fig.1). Of course, the curves with $\mu \neq 0$ go considerably higher, since the bare coupling parameter $g_{\perp}$ decreases with $\mu$.

For $w \neq 0$ the function $1 / g_{e f}(\xi)$ has a minimum both in the AFM and FM cases, position of which, $C_{\min }$, is determined by (A5)-(A7) (in the isotropic case, the minimum occurs in the 3D AFM case only). The minimum may result in non-monotonic temperature dependences of physical quantities which are sensitive to the Kondo effect, e.g., of the effective magnetic moment. The depth of the minimum $\Delta=G_{\min }-G(0)$ is given by

$$
\Delta=\left\{\begin{array}{cc}
\frac{1}{2}[(1+w) \ln (1+w)-w \ln w] & \mathrm{FM} \\
\frac{1}{2}\left[\ln 2+\left(1+w^{2}\right) \ln \left(1+w^{2}\right)-w^{2} \ln w^{2}\right] & 3 \mathrm{D} \mathrm{AFM} \\
\ln \left(w+\sqrt{1+w^{2}}\right) & 2 \mathrm{D} \mathrm{AFM}
\end{array}\right.
$$

Note that at $w \gg 1$ we have in all the cases $\Delta \propto \ln w$.

The critical value $g_{c}$ is determined by the condition

$$
1 / g_{c}=-(1 / \mu) \tanh \left(\mu G_{\min }\right) \simeq\left|G_{\min }-\left(\mu^{2} / 3\right) G_{\min }^{3}\right|, \mu \ll 1
$$

where

$$
-G_{\min }=\left\{\begin{array}{cc}
\lambda+1+\frac{1}{2}[w \ln w+(1-w) \ln (1+w)] & \text { FM } \\
\lambda+\frac{1}{2}\left[1+\ln 2+\ln \left(1+w^{2}\right)\right] & 3 \mathrm{D} \mathrm{AFM} \\
\lambda & 2 \mathrm{D} \mathrm{AFM}
\end{array}\right.
$$

In the FM case anisotropy in $f$-system results in an increase of $g_{c}(|G(0)|$ increases with $w$ more rapidly than $\left.\left|G_{\text {min }}\right|\right)$, in the 3D AFM case $g_{c}$ decreases, and in 2D AFM anisotropy does not influence $g_{c}$. The effective coupling constant $g^{*}=g_{e f}(0)$ remains finite at $g \rightarrow g_{c}-0$ and tends to 


$$
g_{c}^{*}=\mu / \tanh (\mu \Delta)
$$

The effective (renormalized by spin dynamics) Kondo temperature $T_{K}^{*}$ is determined by the condition $1 / g_{e f}\left(-T_{K}^{*}\right)=0$ and satisfies the equation

$$
-G\left(-T_{K}^{*}\right)=\operatorname{arctanh}(\mu / g)
$$

Due to the minimum, $T_{K}^{*}$ is also finite at $g \rightarrow g_{c}+0, T_{K}^{* c}=\left|C_{\min }\right|$.

\section{EFFECTS OF THE F-SYSTEM ANISOTROPY ON SCALING BEHAVIOR}

Now we treat the physically real case $N=2$ with the anisotropy being present in the $f$-system only. To this end we have to consider the full scaling equations for $\mu=0, w \neq 0$. The most important circumstance to be taken into account is the renormalization of the magnon frequencies. Writing down the first integrals of the system (28), (30)

$$
\begin{aligned}
g_{e f}(C)+(2 / a) \ln \bar{\omega}_{e x}(C) & =\mathrm{const} \\
g_{e f}(C)+(2 / b) \ln \omega_{0}(C) & =\mathrm{const}
\end{aligned}
$$

results in

$$
\begin{aligned}
\bar{\omega}_{e x}(C) & =\bar{\omega}_{e x} \exp \left(-a\left[g_{e f}(C)-g\right] / 2\right) \\
\omega_{0}(C) & =\omega_{0} \exp \left(-b\left[g_{e f}(C)-g\right] / 2\right)
\end{aligned}
$$

As follows from (30), (32)

$$
\left(\frac{\bar{\omega}_{e x}(C)}{\bar{\omega}_{e x}}\right)^{1 / a}=\left(\frac{\omega_{0}(C)}{\omega_{0}}\right)^{1 / b}=\frac{\bar{S}_{e f}(C)}{S}
$$

Substituting (49) into (28) we obtain

$$
\partial\left(1 / g_{e f}\right) / \partial \xi=-\eta\left(\exp \left(\xi-\lambda_{e x}-a\left[g_{e f}-g\right] / 2\right), w \exp \left(\xi-\lambda_{e x}-b\left[g_{e f}-g\right] / 2\right)\right)
$$

where

$$
\xi=\ln |D / C|, \lambda_{e x}=\ln \left(D / \bar{\omega}_{e x}\right) \gg 1, w=\omega_{0} / \bar{\omega}_{e x} .
$$


For an antiferromagnet in the nearest-neighbor approximation we have $a=b$ and the equation (51) takes the form

$$
\begin{aligned}
\partial\left(1 / g_{e f}\right) / \partial \xi & \left.=-\Psi\left(\lambda_{e x}-\xi+a\left[g_{e f}-g\right] / 2\right)\right) \\
\Psi(\xi) & =\eta^{A F M}\left(e^{-\xi}, w e^{-\xi}\right)
\end{aligned}
$$

For finite values of $N$ the singularities of the scaling functions, that occur in the magnetically ordered phases, play the crucial role due to peculiar properies of the differential equation (51). In particular, one can prove $\$ 8$ that $g_{\text {ef }}$ diverges at some $\xi$ at arbitrarily small $g$ (i.e. $\left.g_{c}=0\right)$ unless the singularity cutoff is introduced. To make the value of $g_{c}$ finite one has to cut the singularities. This may be performed by introducing small imaginary parts, i.e. by replacing in (A1),(A4)

$$
\begin{aligned}
\ln |1-x| & \rightarrow \operatorname{Re} \ln [1-x(1+i \delta)]=\frac{1}{2} \ln \left[(1-x)^{2}+\delta^{2} x^{2}\right], \\
(1-x)^{-1 / 2} \theta(1-x) & \left.\rightarrow\left\{\left[(1-x)^{2}+\delta^{2} x^{2}\right]^{1 / 2}+1-x\right] / 2\right\}^{1 / 2} /\left[(1-x)^{2}+\delta^{2} x^{2}\right]^{1 / 2}
\end{aligned}
$$

The $x$-dependence of the cutoff parameter can be in principle neglected, as in Ref. [8], since it does not influence appreciably numerical results (since $\delta$ is important at $x=1$ only); however, this dependence is needed to pass correctly to the limit $\omega_{0} \rightarrow 0$ ). As one can see from (27), the value of $\delta$ should be determined by the magnon damping at $q=\left|\mathbf{k}-\mathbf{k}^{\prime}\right| \simeq 2 k_{F}$. This damping is due to both exchange and relativistic interactions. Hereafter we put in numerical calculations $\delta=1 / 100$. We accept also $\lambda=\ln (D / \bar{\omega})=5$.

The dependences $g_{e f}(\xi)$ for 3D FM and AFM phases according to (51), (52) at $g$ close to the critical value are shown in Fig.3. As well as for the isotropic case [8], there occur large intervals of a non-Ferm-liquid (NFL) behavior where magnon spectrum becomes soft. In this regime, the relation between the arguments of the scaling function $\eta(x, y)$ in (51) is fixed by the singularity point $|C|=\bar{\omega}(C)$ (see Appendix, $\bar{\omega}(\xi)$ is defined in the same way as in (41)) or by the condition

$$
\ln [\bar{\omega} / \bar{\omega}(\xi)]=\xi-\lambda
$$


After substituting (49) into (54) we see that the dependence $g_{e f}(\xi)$ is linear in $\xi$ only in the case $a=b$ where

$$
g_{e f}(\xi)-g \simeq 2(\xi-\lambda) / a
$$

Thus for the FM phase the dependence $g_{e f}(\xi)$ in the NFL region is different from the isotropic case.

In the $2 \mathrm{D}$ AFM case the anisotropy does not practically influence the behavior $g_{e f}(C)$ because of strong singularity of the scaling function.

The dependences $1 / g^{*}(g)$ and $\xi^{*}(g)\left(\xi^{*}=\ln \left(D / T_{K}^{*}\right)\right)$ for a 3D anisotropic ferromagnet are shown in Fig.4. These dependences are more similar to those in the isotropic antiferromagnet rather than isotropic ferromagnet (see Fig.5 of Ref. [8]). In particular, a wide plateau with $\xi^{*}(g) \simeq \widetilde{\xi}_{c}^{*}$ can be seen in Fig.4, whereas in the isotropic ferromagnet $\xi^{*}\left(g \rightarrow g_{c}+0\right)$ increases in a not too narrow region. The difference is connected with the absence of the scaling function maximum in the latter case. Thus the anisotropy makes the dependence the effective Kondo temperature on the bare coupling parameter still weaker in comparison with the isotropic case. Due to the minimum of the function $1 / g_{e f}(\xi), g^{*}$ remains finite at $g \rightarrow g_{c}-0$, as well as in the limit $N \rightarrow \infty, g^{*} \rightarrow g_{c}^{*}=1 / \Delta$ with $\Delta$ given by (43). It should be noted that the function $1 / g^{*}(g)$ (Fig.4) approaches $\Delta$ at very small $\left|g-g_{c}\right|$ which are practically unreachable.

On the other hand, strictly speaking, $\xi^{*}(g)$ diverges at $g \rightarrow g_{c}+0$. The increase of $\xi^{*}(g)$ takes place also in an extremely narrow region (of order of $10^{-4}-10^{-3}$ ) only and is not shown in Fig.4. As demonstrate numerical calculations, in this region we have

$$
\xi^{*}(g)-\widetilde{\xi}_{c}^{*} \simeq-\gamma \ln \left[\left(g-g_{c}\right) / g\right], T_{K}^{*} \sim\left(g-g_{c}\right)^{\gamma}
$$

The "critical exponents" $\gamma$ turn out to be non-universal, decreasing with increasing $w$, i.e. the minimum depth; for the 3D ferro- and antiferromagnets with $w=0.3$ we have $\gamma \simeq 0.2$ and $\gamma \simeq 0.05$ (the corresponding values in the isotropic case are $\gamma=1 / 2$ and $\gamma \simeq 0.1$ respectively [8]). 
A comparison of the critical parameter values in the isotropic and anisotropic cases is presented in the Table 1 . One can see that for $N=2$ the anisotropy results in a decrease of the critical value $g_{c}$ in all the cases, unlike the large- $N$ limit. This decrease is more appreciable in the FM phase where linear terms in the anisotropy parameter enter the equations.

Table 1. The critical values $g_{c}$ and $\xi_{c}^{*}$ for different magnetic phases in the isotropic and anisotropic cases at $N=\infty$ (from analytical results, see Sect.4) and $N=2$ (from numerical solution). The parameter values are $\lambda=5, \alpha=1 / 2, \alpha^{\prime}=0$. For $N=2$, the cutoff $\delta=1 / 100$ is used and the "critical value" $\widetilde{\xi}_{c}^{*}$ is estimated from the plateau in the dependence $\xi^{*}(g)$ (see the discussion in the text).

\begin{tabular}{|l|l|l|l|l|l|}
\hline & $w$ & & FM & 3 D AFM & 2D AFM \\
\hline$N \rightarrow \infty$ & 0 & $g_{c}$ & 0.167 & 0.171 & 0.176 \\
\hline & 0.3 & & 0.169 & 0.170 & 0.176 \\
\hline & 0 & $\xi_{c}^{*}$ & $\infty$ & 5.35 & 5 \\
\hline & 0.3 & 5.73 & 5.31 & 5 \\
\hline$N=2$ & 0 & $g_{c}$ & 0.139 & 0.132 & 0.127 \\
\hline & 0.3 & 0.133 & 0.130 & 0.127 \\
\hline & 0 & $\tilde{\xi}_{c}^{*}$ & 6.13 & 6.07 & 6.07 \\
\hline & 0.3 & 6.24 & 6.04 & 6.06 \\
\hline
\end{tabular}

The value of $\widetilde{\xi}_{c}^{*}$ decreases with anisotropy in the AFM case but increases in the FM case. This is explained by that in FM $\omega_{0}$ is renormalized stronger than $\bar{\omega}_{e x}$, so that $\bar{\omega}(\xi)$ tends to zero more rapidly. As demonstrate numerical calculations, $\tilde{\xi}_{c}^{*}$ decreases with $w$ in the AFM case too for sufficiently large $\alpha^{\prime}$.

The experimentally observable quantities can be obtained by using the formulas

$$
T_{K}^{*}=D \exp \left(-\xi^{*}\right)
$$

$\left(g>g_{c}\right)$ and

$$
S^{*}=\bar{S}_{e f}(C=0)=S \exp \left(-\left[g^{*}-g\right] / 2\right)
$$




$$
\begin{gathered}
\bar{\omega}_{e x}^{*}=\bar{\omega}_{e x}(C=0)=\bar{\omega}_{e x} \exp \left(-a\left[g^{*}-g\right] / 2\right) \\
\omega_{0}^{*}=\omega_{0}(C=0)=\omega_{0} \exp \left(-b\left[g^{*}-g\right] / 2\right)
\end{gathered}
$$

$\left(g<g_{c}\right)$. In particular, we obtain the relation

$$
\omega_{0}^{*} / \omega_{0}=\left\{\begin{array}{cc}
\left(S^{*} / S\right)^{2} & \mathrm{FM} \\
S^{*} / S & \mathrm{AFM}
\end{array}\right.
$$

The quantities (58) are finite at $g \rightarrow g_{c}-0$. However, as follows from the standard scaling treatment of the phase transition, in some region we have the law

$$
\bar{\omega}^{*}=\bar{\omega}(C=0) \sim\left(g_{c}-g\right)^{\gamma}
$$

Renormalization of relative anisotropy parameter is given by

$$
w(C)=\frac{\omega_{0}(C)}{\bar{\omega}_{e x}(C)}=\exp \left(-\frac{b-a}{2}\left[g_{e f}(C)-g\right]\right)
$$

This is shown in Fig.5. The corresponding temperature dependence can be obtained by the replacement $|C| \rightarrow T$.

\section{EFFECTS OF ANISOTROPIC $S-F$ COUPLING}

Strictly speaking, in the case where $\mu \neq 0$ the full system of scaling equations cannot be simplified. However, a simple analytical treatment is possible in the case $\mu \ll g$ which is physically real for magnetic systems. Under this condition we can expand

$$
g_{e f}^{\perp}=\sqrt{g_{e f}^{2}-\mu^{2}} \simeq g_{e f}-\frac{1}{2} \mu^{2} / g_{e f}
$$

Provided that the expansion holds at $\xi=0$, this will hold with increasing $g_{\text {ef }}$ too. Then we obtain from (28),(30),(31) the integrals of motion

$$
\begin{aligned}
g_{e f}(C)-\tau \mu^{2} / g_{e f}(C)+(2 / a) \ln \bar{\omega}_{e x}(C) \simeq \text { const, } \tau & =\left\{\begin{array}{c}
1 / 2 \mathrm{FM} \\
-\alpha \mathrm{AFM}
\end{array}\right. \\
g_{e f}(C)-\theta \mu^{2} / g_{e f}(C)+(2 / b) \ln \omega_{0}(C) \simeq \text { const, } \theta & =\left\{\begin{array}{cc}
1 / 2 & \mathrm{FM} \\
-(1-\alpha) s & \mathrm{AFM}
\end{array}\right.
\end{aligned}
$$


so that

$$
\begin{gathered}
\bar{\omega}_{e x}(C)=\bar{\omega}_{e x} \exp \left(-a\left[g_{e f}(C)-g-\tau \mu^{2}\left(1 / g_{e f}(C)-1 / g\right)\right] / 2\right) \\
\omega_{0}(C)=\bar{\omega}_{e x} \exp \left(-b\left[g_{e f}(C)-g-\theta \mu^{2}\left(1 / g_{e f}(C)-1 / g\right)\right] / 2\right)
\end{gathered}
$$

On substituting (65), (66) into (36) we obtain the closed equation

$$
\begin{gathered}
\partial g_{e f} / \partial \xi=-\left(g_{e f}^{2}-\mu\right) \eta\left(\exp \left(\xi-\lambda_{e x}-a\left[g_{e f}-g-\tau \mu^{2}\left(1 / g_{e f}-1 / g\right)\right] / 2\right)\right. \\
\left.w \exp \left(\xi-\lambda_{e x}-b\left[g_{e f}-g-\theta \mu^{2}\left(1 / g_{e f}-1 / g\right)\right] / 2\right)\right)
\end{gathered}
$$

Presence of the terms, that are proportional to $\mu$, in the scaling function arguments in (67), results in a weak smearing of the linear dependence $g_{e f}(\xi)$ in the NFL regime even for $a=b$.

In the FM case, to accuracy accepted, the expressions $(65),(66)$ can be represented as

$$
\begin{aligned}
\bar{\omega}_{e x}(C) & =\bar{\omega}_{e x} \exp \left(-a\left[g_{e f}^{\perp}(C)-g_{\perp}\right] / 2\right) \\
\omega_{0}(C) & =\omega_{0} \exp \left(-b\left[g_{e f}^{\perp}(C)-g_{\perp}\right] / 2\right)
\end{aligned}
$$

Further, as follows from (32), in all the cases

$$
\bar{S}_{e f}(C)=S \exp \left(-\left[g_{e f}(C)-g\right] / 2\right)
$$

Thus the first of relations (50) is violated in AFM case, and the second relation in both FM and AFM cases.

However, the violation owing the anisotropic $s-f$ coupling is weak: renormalization of $\omega_{0}$ for AFM, which is most appreciable, yields in the exponent of (66) the quantity of the order of $\mu^{2} s / g \propto g$ only. Thus the most important effect of $\mu$ is the deformation of scaling trajectories at not too large values of $\xi$, which was considered in Sect.4 (Fig.1), and main corrections to $g_{c}$ are described by (44).

The case of not small $\mu$ (which can be relevant for pseudospin systems [12]) can be investigated by numerical solving the equations (28)- (31). The results are shown in Fig.6. One can see that the anisotropy of $s-f$ coupling leads to an increase of the $g_{c}$ values in comparison with the isotropic case (cf. Table 1). This is due to both decrease of $g_{\perp}$ and more weak renormalization of $\bar{\omega}_{e x}$ according to (30). 


\section{CONCLUSIONS}

In the present paper we have generalized the scaling treatment of Ref. [8] by including the anisotropy in both the $s-f$ coupling and $f$-system itself. We have demonstrated that the magnetic anisotropy modifies considerably the scaling behavior in the Kondo lattice problem.

In all the cases, the anisotropy in the $f$-subsystem (the gap in the magnon spectrum) results in occurrence of a non-monotonous dependence (of a maximum) of the effective coupling constant $g_{e f}(\xi)$. This prevents the increase of $\xi^{*}(g)$ at $g \rightarrow g_{c}+0$, which becomes practically not observable even for a ferromagnet (Fig.4), unlike the isotropic case. The dependence of the effective Kondo temperature on the bare $s-f$ coupling parameter becomes weaker in the presence of the anisotropy. Further, the minimum in the dependence $1 / g_{e f}(\xi)$ results in that $g^{*}$ (and, consequently, $\bar{\omega}_{e x}^{*}, \omega_{0}^{*}$, and $S^{*}$ ) are always finite at $g \rightarrow g_{c}-0$. As for quantitative changes, the anisotropy favors a non-magnetic Kondo state (the critical values of bare coupling constant decrease) The critical region of magnetic instability becomes more narrow (especially in the FM case), so that the non-Fermi-liquid behavior is suppressed. At the same time, anisotropic $s-f$ coupling influences noticeably the form of the scaling trajectory for small $\xi$, but becomes not important with increasing $g_{e f}(\xi)$.

Owing to a more rapid Kondo renormalization of the gap, the system tends to "isotropic" behavior of $g_{e f}(\xi)$ at approaching the strong coupling regime. Such a renormalization (61) may be important for analysis of the spin excitation spectrum in anomalous $f$-systems. One can expect that the observable renormalized spectrum gap in these systems is relatively small and strongly temperature-dependent in comparison with the "usual" $f$-electron magnets. As demonstrate our calculations, next-nearest-neighbor exchange interactions in AFM phase are important for this effect. The dependence $\omega_{0}(T)$ can be investigated not only by the neutron scattering, but also by simple methods like the ferromagnetic resonance.

The change of the critical exponents of the phase transition at $g \rightarrow g_{c}$ with changing the bare anisotropy parameter $w$ turns out to be very strong. It is interesting that their 
values depend continuously on the anisotropy and are non-universal (possibly, higher order contributions to the scaling equations will change this result; this question needs further investigations). It should be noted that, unlike the one-mpurity Kondo problem (where the "phase transition", connected with disappearance of the local moment, exists for $N=\infty$

only, and a crossover takes place for finite $N$, see Ref. [14]), the phase transition in the Kondo lattices is physically real, being a magnetic-nonmagnetic transition. The situation is similar to the onset of magnetism in itinerant electron systems. Of course, one has to bear in mind that the treatment of this transition within the lowest-order scaling may be only qualitatively correct, and a more detailed (e.g., numerical renormalization group) consideration is needed to describe the crossover region.

Similar to Ref. [8], at approaching the critical value of magnetic instability $g_{c}$, the transition to an "incoherent" regime, where non-spin-wave excitations of $f$-system play the dominant role, should be considered. In this regime, the minimum of $1 / g_{e f}(\xi)$ can be suppressed. However, the use of the model "paramagnetic" scaling function for describing the incoherent contribution (as in Ref. [8]) seems to be unreasonable in the presence of the anisotropy, since spin dynamics in the paramagnetic state of $f$-systems in a strong crystal field is rather complicated [9, 15].

The work was supported in part by Grant 96-02-16000 from the Russian Basic Research Foundation.

\section{APPENDIX A: APPENDIX. SCALING FUNCTIONS IN ANISOTROPIC MAGNETS}

Using the long-wave approximations $\omega_{e x}^{F M}(\mathbf{q}) \sim q^{2}, \omega_{e x}^{A F M}(\mathbf{q}) \sim q$ in the whole Brillouin zone (which is justified, e.g., at small $k_{F}$ ), we get from (27)

$$
\eta^{F M}(x, y)=\frac{1}{2 x} \ln \left|\frac{1+x+y}{1-x-y} \frac{1-y}{1+y}\right|
$$

where $\bar{\omega}_{e x}=\omega_{e x}\left(2 k_{F}\right)$. For an antiferromagnet we derive 


$$
\eta^{A F M}(x, y)=\frac{1}{1-y^{2}} \eta^{A F M}\left(\frac{x}{\sqrt{1-y^{2}}}\right)
$$

where $\eta^{A F M}(x)$ is the corresponding scaling function in the isotropic case,

$$
\eta^{A F M}(x)=\left\{\begin{array}{c}
-x^{-2} \ln \left|1-x^{2}\right|, d=3 \\
\left(1-x^{2}\right)^{-1 / 2} \theta\left(1-x^{2}\right), d=2
\end{array}\right.
$$

$\theta(x)$ being the step function. Then we have

$$
\eta^{A F M}(x, y)=\left\{\begin{array}{c}
\frac{1}{x^{2}} \ln \left|\frac{1-y^{2}}{1-x^{2}-y^{2}}\right|, d=3 \\
\frac{\theta\left(1-x^{2}-y^{2}\right)-\theta\left(y^{2}-1\right)}{\left|1-y^{2}\right|^{1 / 2}\left|1-x^{2}-y^{2}\right|^{1 / 2}}, d=2
\end{array}\right.
$$

One can see that the logarithmic singularities of the functions $\eta^{F M}(x)=\eta^{F M}(x, 0)$ and $\eta^{A F M}(x)$ at $x=1$ are shifted to the points $x+y=1(\mathrm{FM})$ and $x^{2}+y^{2}=1(\mathrm{AFM})$, i.e. $|C|=\bar{\omega}$ with $\bar{\omega}$ defined by (41). Besides that, the anisotropy results in occurrence of the second singularity at $y=1$ (i.e., $\left.|C|=\omega_{0}\right)$.

Presence of such singularities is a general property which does not depend on the spectrum model [8]. The functions $\eta^{F M}(x, y)$ and $\eta^{A F M}(x, y)(d=3)$ change their sign at $y(x+y)=1$, i.e.

$$
|C|=\left|C_{\min }\right|=\sqrt{\omega_{0}\left(\omega_{0}+\bar{\omega}_{e x}\right)}
$$

and $x^{2}+2 y^{2}=2$, i.e.

$$
\left|C_{\min }\right|=\sqrt{\omega_{0}^{2}+\bar{\omega}_{e x}^{2} / 2}
$$

respectively. For $d=2$ the function $\eta^{A F M}(x)$ has strong square-root singularities at $x^{2}+y^{2}=$ 1 and $y=1$, and vanishes in the interval $1-x^{2}<y^{2}<1$, i.e.

$$
\left|C_{\min }\right|=\sqrt{\omega_{0}^{2}+\bar{\omega}_{e x}^{2}}>|C|>\omega_{0}
$$

changing its sign after passing this interval (but a smooth contribution occurs for more realistic models of magnon spectrum).

Note that in the limit of strong magnetic anisotropy $\omega_{0} / \bar{\omega}_{e x} \rightarrow \infty$ the singularity at $y \rightarrow 1$ becomes very strong: 


$$
\eta^{0}(y)=\left(1-y^{2}\right)^{-1}
$$

It should be noted that the influence of magnetic anisotropy on spin dynamics can be also considered for the paramagnetic phase (i.e., for the problem of the local moment formation). In this case the singularity (A8) also becomes weaker. If we accept, as in Ref. [8], the "spin diffusion" approximation with the spin spectral density

$$
\mathcal{J}_{\mathbf{q}}(\omega)=\frac{1}{\pi} \frac{\mathcal{D} q^{2}}{\left(\omega-\omega_{0}\right)^{2}+\left(\mathcal{D} q^{2}\right)^{2}}
$$

$\left(\mathcal{D}\right.$ is the spin diffusion constant, $\left.\bar{\omega}_{e x}=4 \mathcal{D} k_{F}^{2}\right)$ we obtain

$$
\eta^{P M}(x, y)=\frac{1}{2 x}\left(\arctan \frac{x}{1-y}+\arctan \frac{x}{1+y}\right)
$$

This function has a finite jump at $|C|=\omega_{0}$. Of course, this approximation can be hardly justified for real $f$-systems. Therefore we do not present concrete calculation results for the PM case. However, one can expect that qualitative effects of anisotropy are similar to those in the magnetically ordered phases.

\section{Figure CAPtions}

Fig.1. The dependence $1 / g_{e f}$ on $\xi=\ln |D / C|$ in the case of anisotropic $s-f$ coupling for a 3D antiferromagnet (upper solid line), 2D antiferromagnet (short-dashed line) and 3D ferromagnet (long-dashed line). The parameters are $\lambda=\ln (D / \bar{\omega})=5, g=0.15, \mu=0.1$. The lower solid line shows the curve for the 3D antiferromagnet with $\mu=0$.

Fig.2. The dependence $1 / g_{\text {ef }}$ on $\xi=\ln |D / C|$ in the presence of anisotropy in $f$-system for a 3D antiferromagnet (solid line), 2D antiferromagnet (short-dashed line) and 3D ferromagnet (long-dashed line). The parameters are $\lambda=5, g=0.15, w=0.3$.

Fig.3. The scaling trajectories $g_{e f}(\xi)$ in an anisotropic ferromagnet for $g=0.1333>g_{c}$ (upper solid line) and $g=0.1332<g_{c}$ (lower solid line), and 3D antiferromagnet for $g=0.1302>g_{c}$ (upper dashed line) and $g=0.1301<g_{c}$ (lower dashed line), $w=0.3$.

Fig.4. The dependences $1 / g^{*}(g)$ for $g<g_{c}$ and $\xi^{*}(g)-\lambda$ for $g>g_{c}$ in an anisotropic ferromagnet with $\lambda=5, \alpha=1 / 2, w=0.3, \delta=1 / 100$. The dashed line is the curve $1 / g-\lambda$. 
Fig.5. The scaling behavior of the effective anisotropy parameter $w_{e f}(\xi)=\omega_{0}(\xi) / \bar{\omega}_{e x}(\xi)$ for an anisotropic ferromagnet with the same parameters as in Fig.2 (solid lines) and antiferromagnet with $\alpha^{\prime}=0.4, g=0.1375>g_{c}$ (upper dashed line) and $g=0.1374<g_{c}$ (lower dashed line).

Fig.6. The dependences $1 / g_{e f}(\xi)$ in the case of anisotropic $s-f$ coupling $(\mu=0.1, w=0)$ for a 3D ferromagnet with $g=0.1619>g_{c}$ (lower solid line) and $g=0.1618<g_{c}$ (upper solid line) and a 3D antiferromagnet with $g=0.1563>g_{c}$ (lower dashed line) and $g=0.1562<g_{c}$ (upper dashed line). 


\section{REFERENCES}

[1] Electronic address: Valentin.Irkhin@imp.uran.ru

[2] G.R.Stewart, Rev.Mod.Phys.56, 755 (1987).

[3] D.T.Adroya and S.K.Malik, J.Magn.Magn.Mat.100, 126 (1991).

[4] C.Lacroix, J.Magn.Magn.Mat.100, 90 (1991).

[5] P.Fulde, J.Keller, and G.Zwicknagl, Solid State Physics, vol.41, ed.F.Seitz et al., New York, Academic Press, 1988, p.1.

[6] G.Zwicknagl, Adv.Phys.41, 203 (1992).

[7] A.M.Tsvelick and P.B.Wiegmann, Adv.Phys.32, 745 (1983).

[8] V.Yu.Irkhin and M.I.Katsnelson, Phys.Rev.B56, 8109 (1997).

[9] P.Fulde and M.Loewenhaupt, Adv.Phys.34, 589 (1986).

[10] P.Nozieres and A.Blandin, J.Phys.(Paris) 41, 193 (1980).

[11] V.Yu.Irkhin, M.I.Katsnelson, and A.V.Trefilov, Phys.Lett.A 213, 65 (1996).

[12] V.Yu.Irkhin, M.I.Katsnelson, and A.V.Trefilov, Europhys. Lett. 15, 649 (1991); Zh.Eksp.Theor.Fiz.105, 1733 (1994).

[13] N.E.Bickers, Rev.Mod.Phys.59, 845 (1987).

[14] P.Coleman, Phys.Rev.B35, 5072 (1987).

[15] S. Maekawa, S.Takahashi, S.Kashiba, and M.Tachiki, J.Phys.Soc.Jpn, 54, 1955 (1985).

[16] T.-S.Kim and D.L.Cox, Phys.Rev.B54, 6494 (1996).

[17] Yu.P. Irkhin, Usp.Fiz.Nauk 151, 321 (1988) [Sov.Phys.-Uspekhi, 31, 163 (1988)].

[18] M.I.Auslender and V.Yu.Irkhin, Z.Phys.B56, 301 (1984). 
[19] V.Yu.Irkhin and M.I.Katsnelson, Phys.Rev.B53, 14008 (1996).

[20] P.W.Anderson, J.Phys.C3, 2346 (1970).

[21] M.I.Auslender and V.Yu.Irkhin, J.Phys.C18, 3533 (1985). 


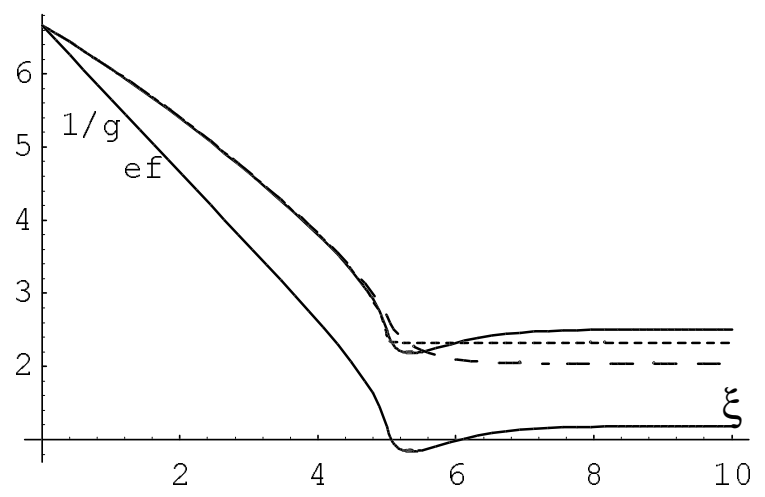




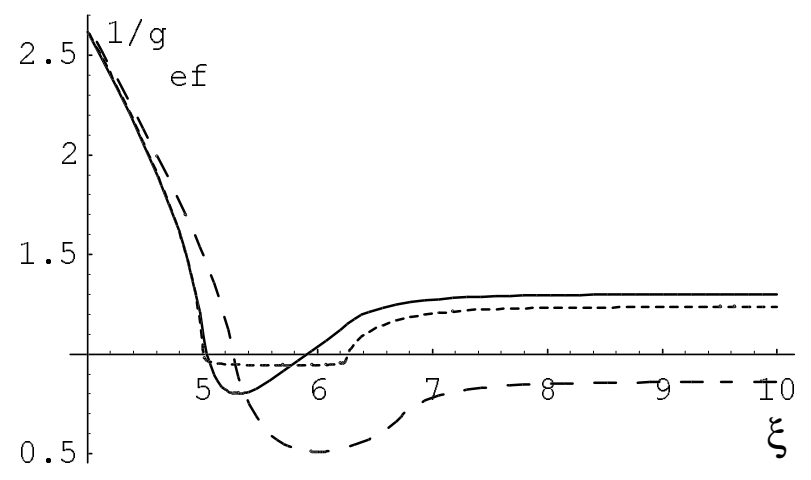




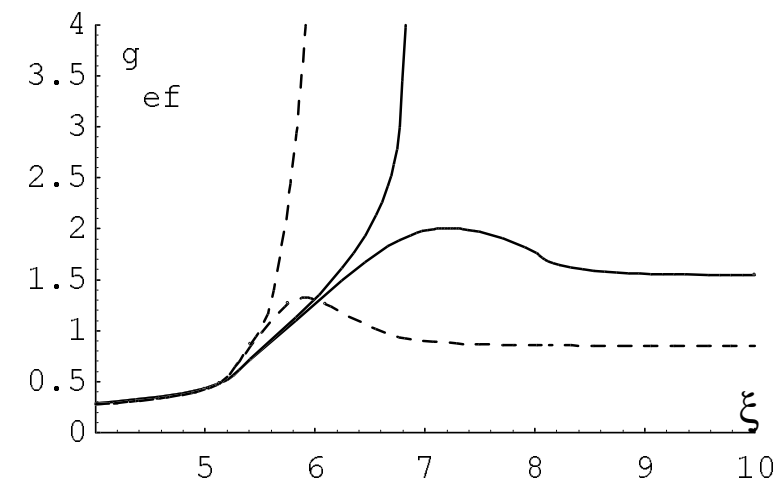




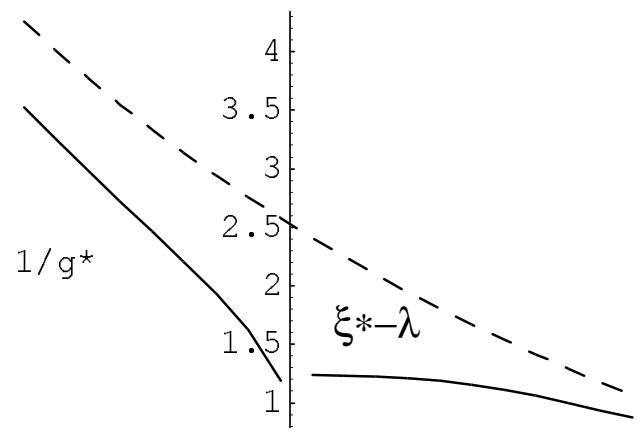

0.105

0.135

0.165 


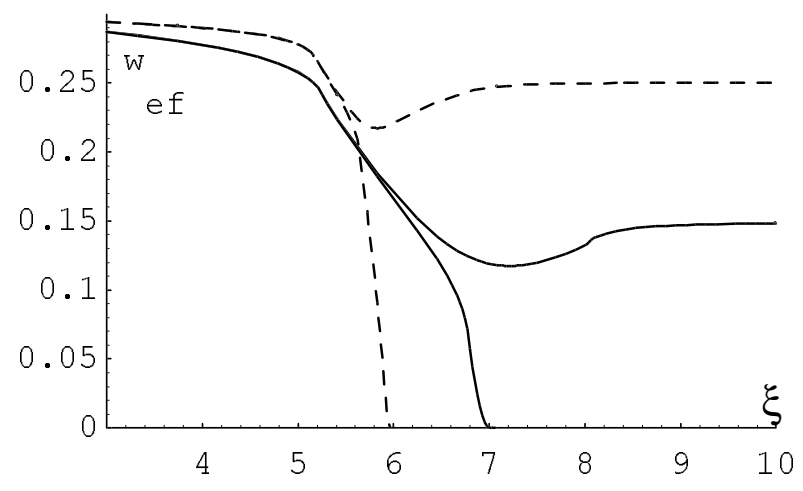




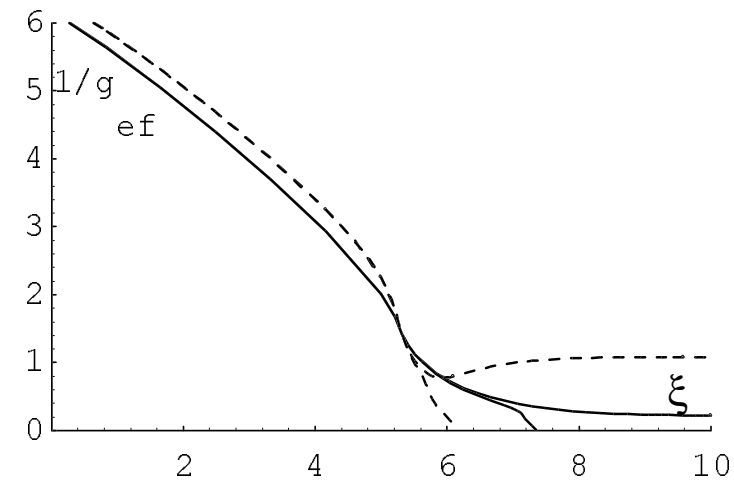

